\title{
A Comparative Study of Outcomes of Type I Tympanoplasty with or without Fibrin Glue
}

\author{
Somu Lakshmanan, ${ }^{1}$ Preethi Umamaheswaran ${ }^{2}$
}

\section{Introduction}

ABSTRACT

Various materials have been used in tympanoplasty to ensure the adherence of the graft with the remnant tympanic membrane. This study aims to compare the clinical outcomes of type I tympanoplasty done with and without fibrin glue.

Materials and Methods

This is a prospective comparative study conducted in a tertiary care centre between August 2014 and July 2016. A sample size of 70 patients was used. The patients were randomly divided into two groups - Group A and Group B. Patients in group A underwent tympanoplasty with fibrin glue and patients in group B underwent tympanoplasty without fibrin glue. The patients were followed up for 6 months and the postoperative hearing improvement and graft uptake rates were compared.

Results

The pre-operative mean pure tone average for group $A$ was $34.33 \pm 7.3 \mathrm{~dB}$; it improved to $22.14 \pm 6.5$ at the end of 6 months. In group $B$, it improved from $34.25 \pm 8 d B$ to $22.64 \pm 7.4 d B$ at the end of 6 months. There was no statistical significance in hearing improvement between both the groups. Though there was no statistically significant difference in the graft uptake rates between group A (94.3\%) and group B (91.4\%), fibrin glue had better outcomes with larger perforations.

\section{Conclusion}

The use of fibrin glue in tympanoplasty is safe and it has a particular advantage in the graft uptake in subtotal perforations. Kevwords

Tympanoplasty; Fibrin Tissue Adhesive

$\mathrm{C}$ hronic Otitis Media - mucosal type is defined as the chronic inflammation of the middle ear cleft in the presence of a persistent tympanic membrane perforation. It is also known as 'safe' ear as life-threatening complications are rare as compared to squamous disease ('unsafe' ear). The treatment of chronic otitis media focuses on the mucosal inflammation in the tympanomastoid compartment. When we are certain that a chronic discharging ear cannot be cured by conservative treatment and have ruled out tubal discharge resulting from nasopharyngeal or sinus suppuration or from allergies, reconstructive middle ear surgery is indicated. ${ }^{1}$

Type I tympanoplasty through a post-aural approach with temporalis fascia is a commonly done procedure. Based on their preference, surgeons employ various techniques like underlay, overlay or inlay. Different materials are also used to ensure the adherence of the graft with the remnant tympanic membrane. The use of fibrin glue is being explored for various otorhinolaryngology procedures, especially in otological surgeries like tympanoplasty.

We have studied the use of fibrin glue (two component fibrin sealant) in type I tympanoplasty and compared it with conventional methods by assessing the audiological outcomes and the graft up-take rates.

\section{Materials and Methods}

This is a prospective comparative study done in the ENT

1 - Department of ENT, Sri Ramachandra Institute of Higher Education and Research Porur, Chennai

2 - Department of ENT, Apollo Specialty Hospital, Chennai Corresponding author:

Dr Preethi Umamaheswaran

email: upreethi@gmail.com 
department of a tertiary care hospital from August 2014 to July 2016. A sample size of 70 patients was randomly divided into two groups - Group A and Group B, using RAS software. All surgeries were performed by a single surgeon. Patients undergoing type I tympanoplasty for chronic otitis media - mucosal type with a dry ear for at least 4 weeks were included in the study. Patients with a sensorineural hearing loss or a conductive hearing loss more than $45 \mathrm{~dB}$ were excluded from the study. Patients undergoing revision surgeries were also not included in the study.

The patients selected as per the inclusion and exclusion criteria were subjected to a detailed history and clinical examination. Otomicroscopy, pure tone audiometry and posterior rhinoscopy were done preoperatively. The size of the tympanic perforation was noted and based on the number of quadrants involved and were divided into small, medium, subtotal and total perforations. ${ }^{2} \mathrm{~A}$ written informed consent was obtained from the patient.

The surgery was done under general anaesthesia. As a standard, temporalis fascia graft was harvested by postaural route and was used in the repair of the perforation. The procedure was done under the vision of an operating microscope.

- In Group A, fibrin glue is applied along the edges of the perforation.

- In Group B, fibrin glue is not applied.

Following the procedure, the external auditory canal was packed with gelfoam and the postaural wound was closed in layers with 3-0 vicryl (Polyglactin 910) and 3-0 ethilon (monofilament nylon) following which sterile dressing was applied.

The patients were followed up for 6 months. Postoperatively, the graft uptake was assessed at the end of one month, three months and six months. Pure tone audiometry was done postoperatively at three and six months.

\section{Results}

A total of seventy patients were included in the study who underwent type I tympanoplasty between August, 2014 and July, 2016. They were randomly divided into two groups. In one group fibrin glue was used (Group A) and in the other group fibrin glue was not used (Group B).

There were 10 right ears and 25 left ears in group A and there were 11 right ears and 24 left ears in group B. There was no statistically significant difference between the two groups.

In group $\mathrm{A}$, there were 11 subtotal perforations, 7 involving the antero-inferior and antero-superior quadrants, 5 involving the antero-inferior and posteroinferior quadrants, 4 involving the postero-superior and postero-inferior quadrants, 5 involving the anteroinferior quadrant alone and 3 involving the posteroinferior quadrant alone.

In group $\mathrm{B}$, there were 11 subtotal perforations, 2 involving the antero-inferior and antero-superior quadrants, 7 involving the antero-inferior and posteroinferior quadrants, 4 involving the postero-superior and postero-inferior quadrants, 7 involving the anteroinferior quadrant alone and 4 involving the posteroinferior quadrant alone.

\section{Hearing improvement:}

The pre-operative mean pure tone average for group A was $34.33 \pm 7.3 \mathrm{~dB}$; it improved to $24.48 \pm 8 \mathrm{~dB}$ at the end of 3 months and to $22.14 \pm 6.5$ at the end of 6 months. There was a statistically significant improvement in the hearing post-operatively.

The pre-operative mean pure tone average for group $B$ was $34.25 \pm 8 \mathrm{~dB}$ which improved to $25.25 \pm 8.7 \mathrm{~dB}$ at the end of 3 months and to $22.64 \pm 7.4 \mathrm{~dB}$ at the end of 6 months. There was a statistically significant improvement in the pure tone average post-operatively.

\section{Graft uptake:}

Of the 70 patients who underwent type I tympanoplasty in this study, 65 patients had the graft in-situ at the end of six months. Two patients in group A had perforations at the end of six months and two patients in group B had a medialised graft and one had a perforation.

Group A had an overall graft up-take rate of $94.3 \%$ whereas group B had a graft up-take rate of $91.4 \%$. 


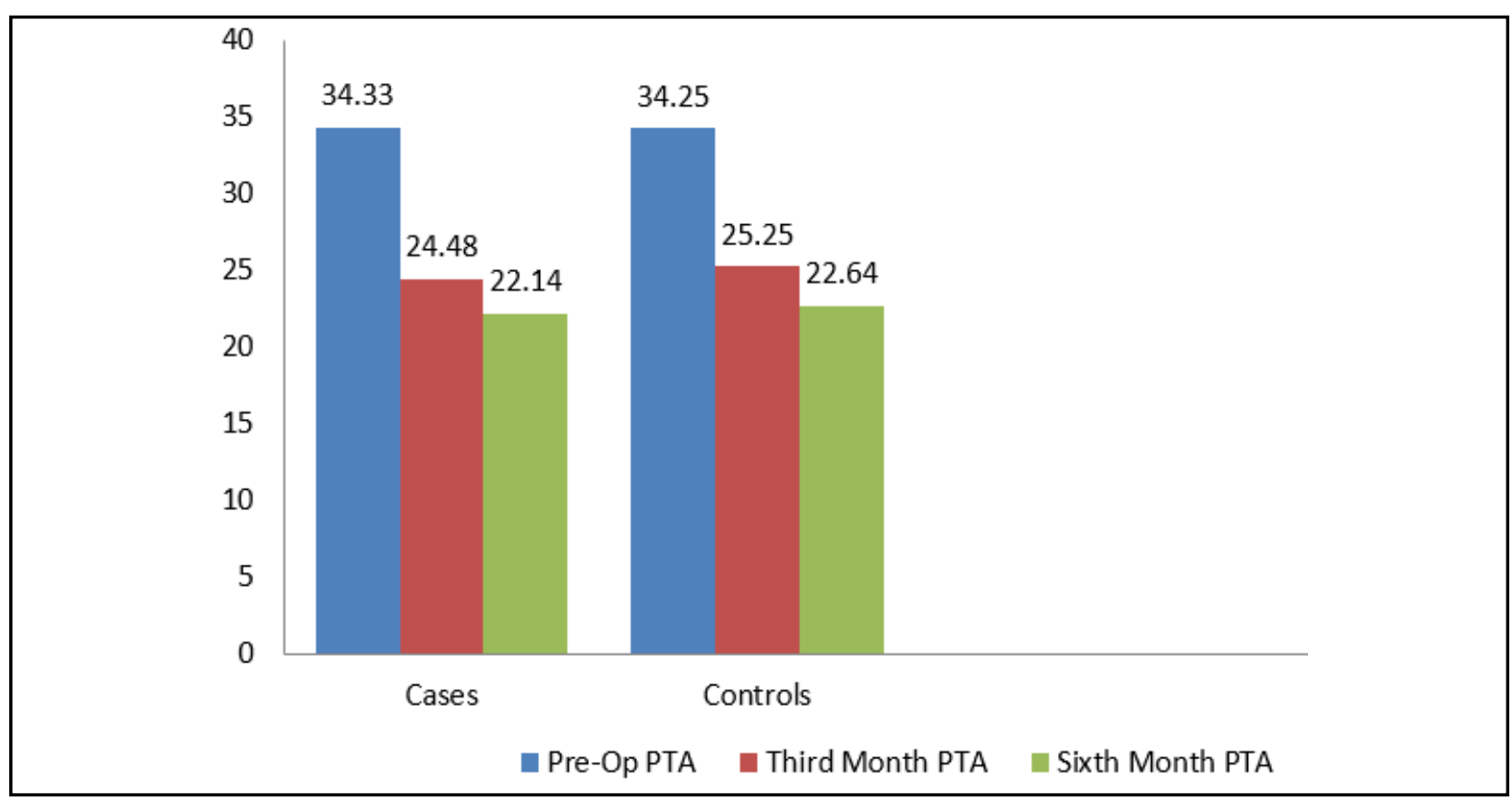

Fig. 1. Comparison of postoperative PTA

\section{Discussion}

The aim of our study was to assess and statistically analyse the hearing improvement and graft up-take in type I tympanoplasty done with fibrin glue as compared to conventional techniques. A total of 70 patients were divided randomly into two groups (A and B) of 35 each using RAS software.

The standard procedure used in this study was type I tympanoplasty done via a post-aural approach with a temporalis fascia graft by underlay technique. The placement of the fibrin glue was done after the placement of the graft and repositioning the tympanomeatal flap, along the edges of the perforation. Similar method of placement of graft and application of fibrin glue was done by Yuasa et al and Sakagami et al. ${ }^{3,4}$ But Lai and Propst in their study placed the graft lateral to the tympanic membrane and used fibrin glue to secure the graft. $^{5}$

None of our patients developed allergic reaction to the fibrin glue. We observed that there was a statistically significant improvement ( $p$ value $=0.0000$ ) in the pure tone average post-operatively when recorded at the third month and a further improvement though minimal was noted in the pure tone average at the sixth month in both the groups. In some cases the pure tone average worsened at the sixth month when compared to the third month; this could be associated with the subjective nature of the pure tone audiometry test as there were no obvious signs noted on examining the ears of these patients.

A mean hearing improvement of $12.19 \mathrm{~dB}$ was noted in group A while in group B, a hearing improvement of $11.61 \mathrm{~dB}$ was noted. Similar hearing improvement was reported by Yuasa et al. ${ }^{3}$ Maeta et al published their results comparing simple myringoplasty done with and without fibrin glue and inferred that the simple method with fibrin glue had the advantage of good hearing prognosis over orthodox myringoplasty. ${ }^{6}$ But in our study though the improvement in hearing with fibrin glue was better it was not statistically significant.

We recorded a graft up-take rate of $94.3 \%$ in group A and $91.4 \%$ in group B. Similar graft up-take rates were observed by Yuasa et al and Lai \& Propst in their studies. ${ }^{3,5}$ Maeta et al had a graft up-take rate of $90.8 \%$ in 109 patients who underwent orthodox myringoplasty and $79.8 \%$ in 84 patients who underwent simple myringoplasty with fibrin glue. ${ }^{6}$ Though in our study 
the conventional group had a comparable graft up-take rate to Maeta's study, the graft up-take rate in the fibrin glue group is significantly less compared to our study as well as the other studies mentioned above. Masayuki et al. have recorded a graft uptake of $91 \%$ in patients undergoing myringoplasty with fibrin glue. ${ }^{7}$ However that study had a sample size of 11 patients only.

In our study there were 11 subtotal perforations in both the groups. In group A all 11 had successful graft up-take $(100 \%)$ at the end of six months. Whereas in group B 2 out of the 11 cases presented with a medialised graft leading to a graft up-take rate of $81.8 \%$. Thus, fibrin glue has a particular advantage in large perforations. Fitzgerald et al observed that fibrin glue helped in fixing of fascia grafts in subtotal perforations during tympanoplasty. ${ }^{8}$ Maeta et al observed that though the overall graft up-take was poor compared to orthodox myringoplasty, the use of fibrin glue in large perforations produced better hearing outcomes and better graft uptake rates.

Though the use of fibrin glue has no effect on hearing improvement in the postoperative period, it helps in securing the graft following tympanoplasty.

\section{Conclusion}

The use of fibrin glue in type I tympanoplasty is not an extensively studied subject. The graft up-take rates were better in group A (94.3\%) compared to group B $(91.4 \%)$ at the end of six months, though the difference was not statistically significant. None of the patients in our study had an adverse allergic reaction to the fibrin glue. Hence the use of fibrin in middle ear surgeries is safe and can be used without fear of adverse reactions. The cost of fibrin glue prevents its incorporation into the standard procedure of type I tympanoplasty but it provides an advantage in securing the graft in patients with large perforations and hence should be considered in patients with subtotal perforations.

\section{Reference}

1. Proctor B. A Statistical Review of 177 Tympanoplasties. A.M.A. Archives of Otolaryngology 1960; 71:123-31

2. Saliba I. Hyaluronic acid fat graft myringoplasty: how we do it. Clinical Otolaryngology 2008; 33: 610-4. doi:10.1111/j.17494486.2008.01823.x

3. Yuasa R, Saijo S, Tomioka Y et al. Office closure of eardrum perforation with fibrin glue (in Japanese), Otolaryngol Head Neck Surg (Tokyo) 1989; 61:1117-22

4. Sakagami M. Simple underlay myringoplasty. The Journal of Laryngology and Otology 2007; 121:840-4

5. Lai P, Propst EJ. Lateral graft type I tympanoplasty using alloderm for tympanic membrane reconstruction in children. International Journal of Pediatric Otolaryngology 2006; 70: 1423-9

6. Maeta M, Saito R, Nakagawa F, Miyahara T et al. A clinical comparison of orthodox myringoplasty and a simple method with fibrin glue. Nippon-Jibiinkoka- Gakkai-Kaiho 1998; 101:1062-8

7. Furukawa M, Hayashi C, Narabayashi O et al. Surgical Management of Myringosclerosis over an Entire Perforated Tympanic Membrane by Simple Underlay Myringoplasty. International Journal of Otolaryngology, vol. 2016, 2016. https://doi.org/10.1155/2016/2894932

8. O'Connor F, Shea JJ. A biologic adhesive for otologic practice. Otolaryngol Head Neck Surg. 1980; 90: 347-8 